\title{
Modulation transfer of the human eye as a function of retinal eccentricity
}

\author{
Rafael Navarro and Pablo Artal \\ Instituto de Optica, Consejo Superior de Investigaciones Científicas, Serrano 121, 28006 Madrid, Spain \\ David R. Williams \\ Center for Visual Science, University of Rochester, Rochester, New York 14627
}

Received March 30, 1992; revised manuscript received July 20, 1992; accepted July 20, 1992

\begin{abstract}
We measured the monochromatic image quality of the eye across a wide visual field $\left(120^{\circ}\right)$, with natural pupil $(4 \mathrm{~mm})$ and accommodation (3 diopters). The method is based on the acquisition and the posterior processing of double-pass aerial images of a point source imaged on the retina, which was kept at a fixed distance from the eye at all retinal eccentricities. The two-dimensional modulation transfer functions (MTF's) computed from the aerial images show that astigmatism is the dominant monochromatic aberration in both the fovea and the periphery and is also the major cause of variability among individuals. We found a slower decline in optical quality with eccentricity than had been found by previous measurements. Our foveal results are in close agreement with those of Campbell and Gubisch [J. Physiol. (London) 186, 558-578 (1966)], but off-axis optical quality is much better than found previously by Jennings and Charman [Am. J. Optom. Physiol. Opt. 55, 582-590 (1978); Vision Res. 21, 445-454 (1981)]. The optical system of the eye seems to follow a wide-angle lens design: the optical quality in the center (fovea) is not particularly good (it is far from the diffraction limit at this pupil size), but the modulation transfer function remains roughly constant for a wide visual field.
\end{abstract}

\section{INTRODUCTION}

Many experimental studies have been published on the optical quality of the eye, from measurements of geometrical aberrations ${ }^{1}$ to more recent measurements of linespread functions, ${ }^{2-4}$ point-spread functions ${ }^{5,6}$ (PSF's), and modulation-transfer functions ${ }^{7-10}$ (MTF's). Image quality in the fovea has been widely studied for different pupil diameters, ${ }^{9}$ as a function of focus, ${ }^{11}$ and with different methods: double-pass objective techniques, psychophysical interferometric methods, ${ }^{12}$ and computations from wave aberrations. ${ }^{13-14}$ However, little attention has been paid to the peripheral optical quality of the eye, and most studies have been restricted to measurements of the offaxis refractive state. ${ }^{15-17}$ Only Jennings and Charman ${ }^{18,19}$ have reported data on peripheral optical quality. Their results typically showed lower optical quality in the fovea than that shown by most other measurements. Recently image quality was studied at small eccentricities ${ }^{6}$ and at the optic axis ${ }^{12}$ (typically $5^{\circ}$ away from the fovea). In both cases the MTF's were found to be close to, but slightly below, those obtained at the center of the fovea.

In this paper we present experimental results of the monochromatic image quality of the eye across a wide $\left(120^{\circ}\right)$ visual field. Our experiment was performed with a natural pupil $(4 \mathrm{~mm})$ and free accommodation [fixed viewing distance, 3 diopters (D)]. In measurements of optical quality it is sometimes convenient to paralyze accommodation and use artificial pupils in order to avoid fluctuations in both accommodation and pupil diameter. Further investigations of the double-pass method itself, using paralyzed accommodation and artificial pupils, will be published elsewhere. Arnulf and co-workers ${ }^{20}$ developed a technique in which the double-pass aerial image of a point source was recorded dynamically with an image intensifier. That allowed the aerial PSF and the effect of the microfluctuations of accommodation to be visualized in real time. Subsequently an optical-digital method $^{5}$ was implemented that was based on the recording of short-exposure images with a linear detector; this method has since been further improved ${ }^{6,12}$ and permits the measurement of the MTF in natural viewing conditions (with the lens in its natural shape). It also avoids the alignment problems that are associated with the use of artificial pupils.

Our experiments use monochromatic light, so the resulting MTF's do not include the effect of chromatic aberration, ${ }^{21-23}$ which is an important source of blur when the retinal image is polychromatic.

\section{EXPERIMENTAL METHOD}

\section{A. Apparatus}

The apparatus used to measure the MTF is a modified and improved version of that previously developed by Santamaría et al., ${ }^{5}$ as depicted in Fig. 1: a He-Ne laser (nominal power $10 \mathrm{~mW}$ ) is used as a coherent light source. The light beam passes first through a neutral-density filter, LND, which reduces the light intensity to a value that is safe for the eye. It also reduces the intensity on the CCD that records the aerial image to a range that avoids saturation. A second neutral-density filter, ND, with a density of $4 \mathrm{log}$ units, is mounted upon a rotary solenoid, $\mathrm{RS}$, so that it can be moved in and out of the beam. This filter serves as a shutter and is synchronized with the 
CCD camera. The observer can use the point source, $O$, as a foveal fixation target, since it is visible even with the filter in place. The beam is spatially filtered by a $20 \times$ microscope objective, $\mathrm{MO}$, and a $10-\mu \mathrm{m}$ pinhole, $\mathrm{O}$, which acts as the point test. The point source actually subtends 0.17 arcmin, which is more than five times smaller than the eye's foveal PSF, so its finite size can be ignored. The emerging beam is collimated by lens $L_{1}\left(f^{\prime}=200 \mathrm{~mm}\right)$, and approximately $8 \%$ of this light is reflected by a pellicle beam splitter, PBS. The remaining light is transmitted through the pellicle and is eliminated from the system by a black diffuser, BD. The beam is focused by lens $\mathrm{L}_{2}$ $\left(f^{\prime}=300 \mathrm{~mm}\right)$ at a distance corresponding to the inner spherical surface of a perimeter. The beam passes through a hole in the perimeter in transit to the eye. For off-axis measurements, a circular, red spot projected onto the perimeter surface, $\mathrm{C}$, serves as a fixation and accommodation target (ticks and marks made on the perimeter surface for reference also aid accommodation). This test is viewed binocularly at a fixed distance corresponding to the perimeter radius, which is equivalent to $3 \mathrm{D}$ of accommodation (near vision). In the foveal case, the point test serves also as a fixation and accommodation target. Although this point alone would not be a good one to maintain accommodation, the sharp edge of the circular hole in the perimeter, which subtends $3^{\circ}$ of the visual field, is a strong stimulus to accommodation.

The optical system of the eye forms the image of the object $\mathrm{O}$ on the retina, $\mathrm{O}^{\prime}$. A small fraction of the light of the incident beam is reflected by the retina; this light passes again through the ocular media and lens $\mathrm{L}_{2}$ and also through beam splitter $\mathrm{PBS}_{2}$. Finally, the zoom lens, $\mathrm{Z}\left(f_{z}^{\prime}=60-300 \mathrm{~mm}\right)$, forms the aerial image, $\mathrm{O}^{\prime \prime}$, of the retinal image $\mathrm{O}^{\prime}$ on a $C C D$ videocamera. The zoom controls the magnification of the aerial image from $3.6 \times$ to $18 \times$ (given by the ratio of the power of the eye to that of the zoom). This allows us to adjust the physical size of the aerial PSF, which increases with eccentricity, to fit within the camera viewing window.

The videocamera (Hitachi solid-state KP-140), along with the frame grabber (MATROX MVP-AT imageprocessing board), has been calibrated, providing a linear response. The size of the pixels is $13.23 \mu \mathrm{m}$ (horizontal) $\times 11.35 \mu \mathrm{m}$ (vertical). However, the final effective resolution of the camera-frame-grabber system is not given by the pixel size; factors such as the interlaced scan of the video signal, which makes the real vertical resolution equivalent to 2 pixels, make it necessary to oversample the aerial image $\mathrm{O}^{\prime \prime}$ by at least a factor of 2 . For small eccentricities we have used a zoom focal length of $200 \mathrm{~mm}$, which corresponds to a theoretical sampling frequency of 156 cycles per degree (cpd). However, the real maximum measurable frequency is $156 / 2=78 \mathrm{cpd}$ in the vertical direction and is some intermediate value (approximately $100 \mathrm{cpd}$, owing to electronic signal-processing limitations) in the horizontal direction.

\section{B. Pupil Size Control}

To perform the experiment with a natural pupil, one must monitor and control the pupil size. For this purpose an intensified TV camera (image intensifier, lens, and CCD camera) displays the image of the pupil on a TV screen with a calibrated reticle. The pupil size can be measured in this way with an accuracy of approximately $0.25 \mathrm{~mm}$. The intensifier also allows the experimenter to see simultaneously which part of the eye is illuminated by the laser beam, thus permitting pupil centering. In order for the eye to be aligned for a given eccentricity, the subject's head is first rotated to the fixation angle and then is adjusted horizontally until the pupil of the eye is in the center of the beam. Although the position of the natural pupil is not critical, it is important to avoid any possible vignetting. The experimenter controls the pupil size by varying the luminance of the perimeter screen (rejecting the frames when the pupil size differs more than $0.25 \mathrm{~mm}$ from the desired value). The subject delivers the light flash when he or she is fixating and accommodating properly.

\section{Photometry and Eye Safety}

The exposures that we used produced a maximum laser irradiance in the pupil plane of approximately $0.3 \mathrm{~mW} / \mathrm{cm}^{2}$. For a 4-mm pupil diameter, the laser power entering the eye is less than $0.04 \mathrm{~mW}$. This is a worst-case value, for large eccentricities when the intensity of the aerial image is widely spread. Since the exposure time is of the order of 60-100 ms, our most intense exposures are approximately $1 / 50$ or less of the maximum limit allowed by American National Standards Institute safety standards. ${ }^{24}$ For example, the standard maximum level permitted for looking directly into a red $\mathrm{He}-\mathrm{Ne}$ laser beam is $2 \mathrm{~mW}$ for an exposure time of $0.25 \mathrm{~s}$, which is roughly the reaction time for blinking.

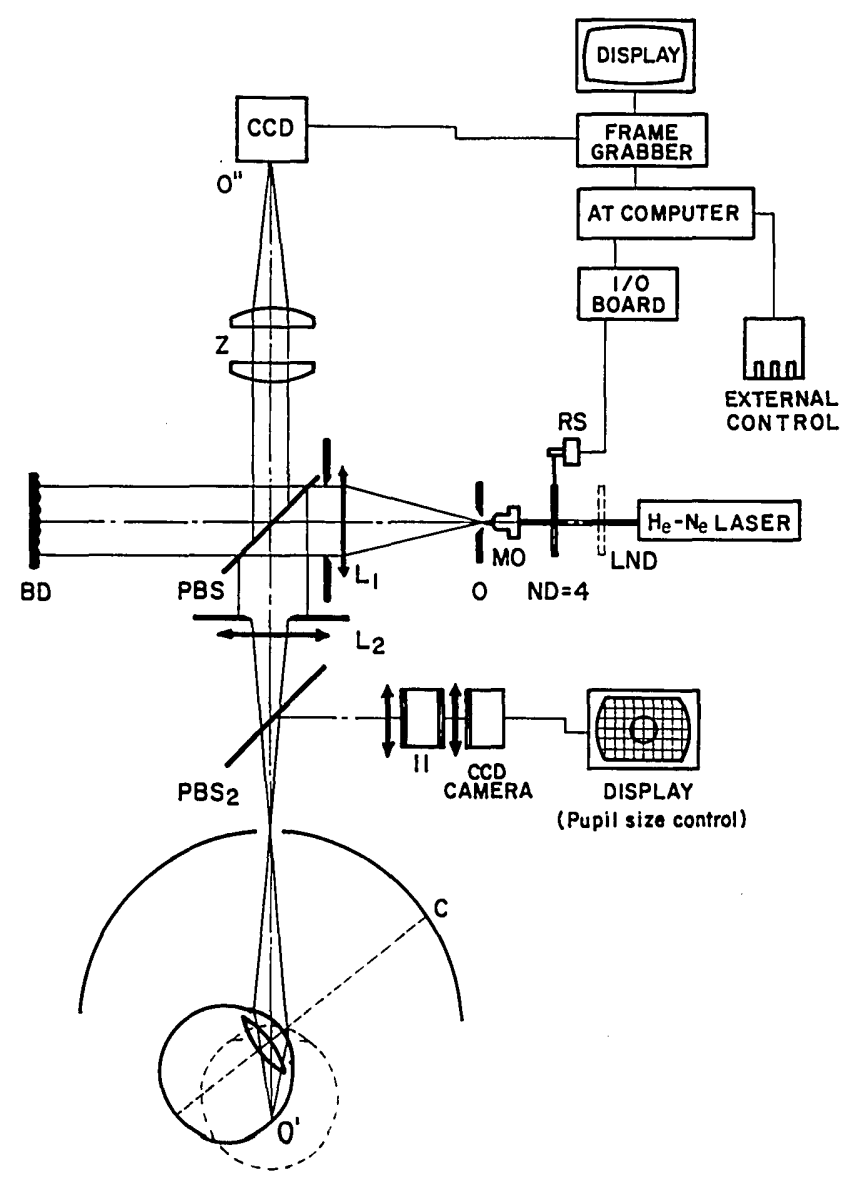

Fig. 1. Experimental setup for recording and digital processing of the double-pass aerial image of a point source (see text for a detailed description). 


\section{Computation of the Modulation Transfer Function}

The double-pass method for obtaining the optical transfer function of the eye is based on two main assumptions, which have been discussed elsewhere. One assumption is that the retina acts as a diffusing surface, which produces the appearance of speckle in the short-exposure aerial image but does not significantly degrade image quality. ${ }^{6,12}$ The other assumption is that the second pass through the ocular media is incoherent. This assumption is required for obtaining the retinal PSF from the aerial image by autodeconvolution. ${ }^{5,6}$ Although the second pass is coherent for each short exposure, averaging a number of shortexposure frames makes it essentially incoherent. Both computer simulations and experimental measurements suggest that averaging 32 independent (uncorrelated) short-exposure aerial images is sufficient to remove most speckle noise, i.e., coherence. Consequently one of the steps of our image-processing procedure is to average 32 short-exposure images.

The frames are corrected for both bias and pixelsensitivity differences (flat field), which are the two main sources of systematic photometric errors in CCD cameras. For this purpose a bias image is automatically acquired an instant before the ND filter is removed and is then subtracted from the frame. The result is divided (pixel by pixel) by a flat-field white image, which has been grabbed previously. Our frame grabber has an 8-bit/pixel (256 levels) analog-to-digital converter. In order to avoid saturation we impose an upper limit, 225, for the maximum intensity level permitted. We also impose a lower threshold for the maximum pixel-intensity value, 80 (roughly one third of the maximum). Since defocusing causes a steep decline in the maximum intensity of the double-pass PSF, this threshold also imposes an indirect, but accurate, further control on the focus of the eye. The fraction of the frames that are accepted can vary considerably (depending mainly on the degree of cooperation and self-control of the subject), but on average it was more than $50 \%$, and it was more than $80 \%$ in many cases.

Once we have obtained an aerial image and previewed it to determine its acceptability, we perform the following image-processing procedure. First, the centroid of the aerial image is computed, and a processing window of $256 \times 256$ pixels centered on the aerial image centroid is set. This image is added to previously acquired images, collected under the same conditions, until 32 images have been obtained. This composite aerial image shows practically no speckle; since individual speckles are different in each snapshot, they are blurred in the sum of all 32 images.

Ideally, one would like to collect only the light that passes into the photoreceptors, is reflected from the pigment epithelium at the end of the outer segments, and remains in the photoreceptors as it travels back toward the pupil of the eye. When entering the eye, this waveguided light follows a path that is most similar to the light actually used for seeing. But the aerial image contains light that is returning from many sources besides the photoreceptors, including stray light within the optical system itself as well as light reflected from other ocular structures (e.g., the cornea, the inner limiting membrane, and the choroid). ${ }^{25}$ At present there is no way to isolate the waveguided component, although evidence that the returned light is strongly directional ${ }^{26,27}$ implies that a major fraction of that light is waveguided by photoreceptors. There is some evidence that the appropriate choice of wavelength $^{11}$ or polarization ${ }^{10}$ can increase this fraction, but we have not explored these possibilities in the present study. In the absence of a secure and complete theoretical model of fundus reflectance, or of a way to obtain a direct characterization of all the unwanted components (across the visual field), we have adopted the following procedure, which is in essence the same as that adopted by previous practitioners of the double-pass method.

The aerial image typically contains a dim, nearly uniform background. We estimate this background from the average intensity at the four corners of the processing window. We then subtract this estimate from the image. We do this on the assumption that the unwanted light is more uniformly distributed because it arises from diffuse reflections, or layers that are defocused with respect to the photoreceptor layer. Certainly this assumption is not completely accurate. For example, it neglects specular reflection from the inner limiting membrane, which at least in the fovea is only slightly defocused. Furthermore, we know from psychophysical studies of glare that the singlepass retinal PSF contains some light far out in the tails of the light distribution. ${ }^{28}$ Although this light is so dim that its contribution to the MTF is negligible (in young subjects), ${ }^{29}$ the aerial image cannot be strictly zero at the edge of our window. Simon and Denieul ${ }^{30}$ showed that the double-pass MTF depends on how much of the light in the tails of the aerial image is collected. Therefore the resulting MTF depends on the window size, or field of view.

In our experiments there are two ways in which the aerial images may have been truncated, from vignetting by the pupil of the eye and from the finite size of the CCD array. In the far periphery $\left(60^{\circ}\right)$ the pupil truncated the aerial image. This could have an important effect, since the angle that is subtended by the pupil is approximately the same as the angle that is subtended by the large aerial image. With the low magnification that is used with large eccentricities, the pupil acts as a slightly defocused field stop. This truncation effect can be seen clearly in the images corresponding to $60^{\circ}$ in Fig. 2, in the way in which the lower contour lines tend to follow the circular shape of the digital window. This effect is also visible at $50^{\circ}$ (for subject PA, and much less for subject SB). In these cases, the window is clearly vignetting the tails of the aerial images, which causes a systematic overestimation of the MTF. In principle, we could have avoided this kind of vignetting problem by placing the camera lens one focal length from an image of the pupil. However, this would have required placing additional lenses in front of the eye and was not done. Since pupil vignetting is important only in the far periphery, we have preferred not to modify our apparatus. One of the most important advantages of our system is that it permits natural accommodation by binocular viewing of the perimeter screen with the naked eye.

The limited field of view of the camera always creates a similar effect, vignetting somewhat the tails of the aerial image. We adjusted our zoom magnification to fit the size of the aerial image in the camera's field of view, but the limited size of the CCD produces vignetting that can be large in some cases (typically at $20^{\circ}$ and $50^{\circ}$, i.e., before the image passes to a further reduction in magnification). 


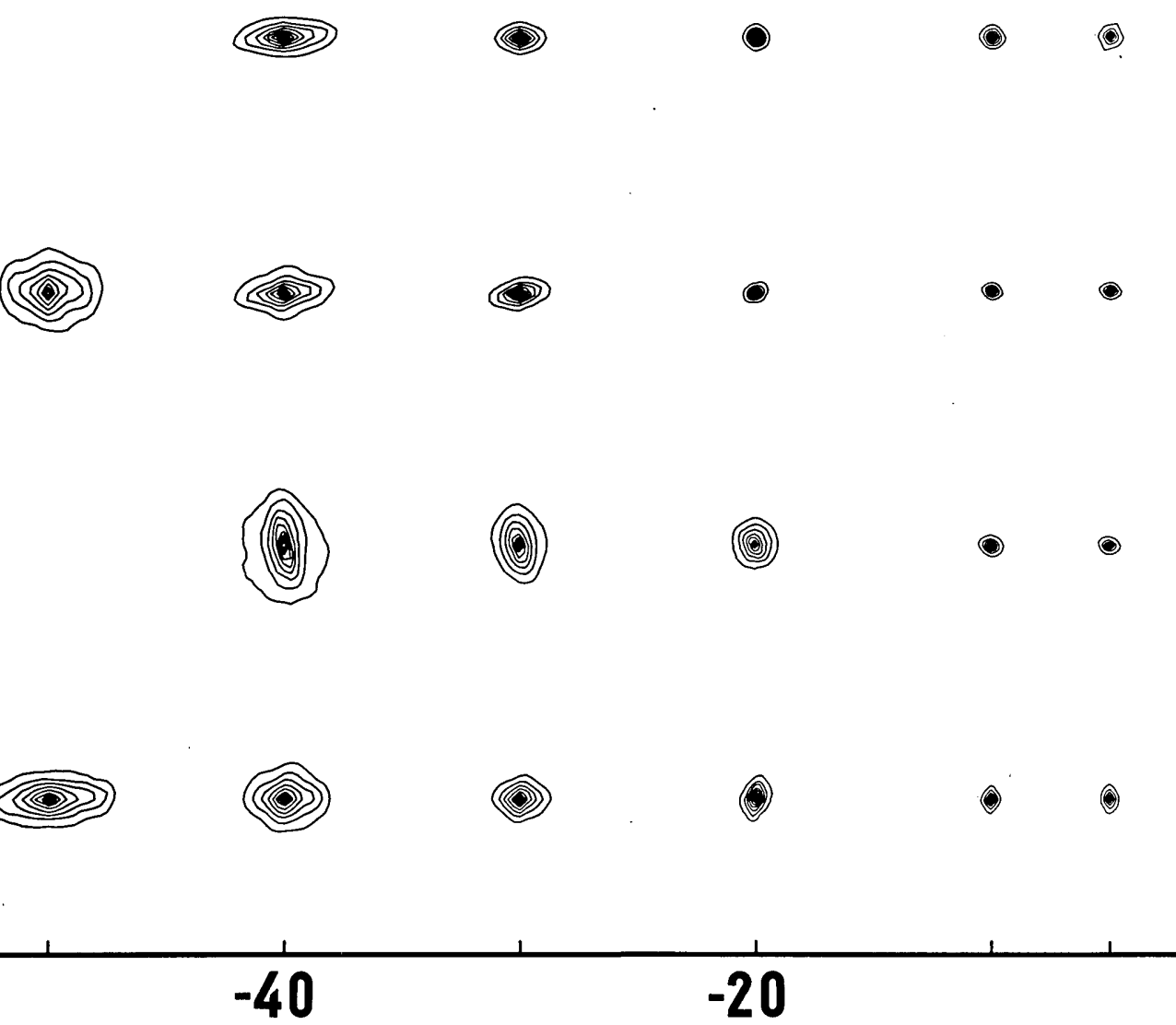

DEG.

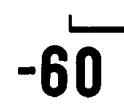

$-20$

Fig. 2. Continues facing page.

In the periphery, whereas the central core of the image is still small (high resolution), the tail can be more spread and relatively brighter, so vignetting problems are more likely. This effect is too small to be noticed in the aerial images (except in the far periphery), but some features of the MTF's radial-profile family of curves could be a sign of the presence of vignetting. (In Fig. 9 below, the two straight parts - low- and high-frequency exponentials - of the curves show different patterns of change with eccentricity. The low-frequency part follows a well-behaved gradual increase of slope, while the slope of the secondhigh-frequency-exponential decreases in two cases from $10^{\circ}$ to $20^{\circ}$ and from $40^{\circ}$ to $50^{\circ}$. This decrease may indicate a vignetting effect: overestimation of the MTF in the high-frequency range.) Vignetting has also been confirmed experimentally, being the major effect of the window. Although we are currently working on a more rigorous treatment of this problem, for now we believe that our present procedure provides an estimate of the single-pass retinal MTF that is not grossly distorted. In fact our foveal data are in reasonably good agreement with psychophysical single-pass MTF's estimated as the ratio of coherent to incoherent contrast sensitivity, measured in the same observers with the same pupil size and wavelength. ${ }^{12}$

We correct the aspect ratio $x / y$ of the pixels, which is approximately $6 / 5$ as a result of the 60/50 European/ American hertz ratio, by computing in each row (along the $x$ axis) the new samples by interpolation. Finally, we apply a circular window (256 pixels in diameter) with smooth Gaussian edges to the aerial image in order to avoid possible edge effects in the Fourier transform.

Next we compute the square root of the modulus of the Fourier transform of the aerial image. Applying the square root in the frequency domain is equivalent to deconvolving the aerial image, which has been blurred twice by the optics of the eye. The phase of the optical transfer function ${ }^{31}$ is typically quite flat for this pupil size, so it has not been considered in this study. A further white-noiseremoval procedure consists of subtracting the local average value in the four corners of the two-dimensional MTF window. Since the spatial frequencies in the corners are beyond the diffraction limit, we know that the signal must be zero; thus all the energy there is noise. This value is typically less than $1 \%$ of the center.

\section{E. Experimental Procedure}

We obtained the average double-pass aerial image and computed the MTF in 15 retinal locations along the horizontal meridian in four normal young subjects with small amounts of ametropia in the fovea: 
OEG.

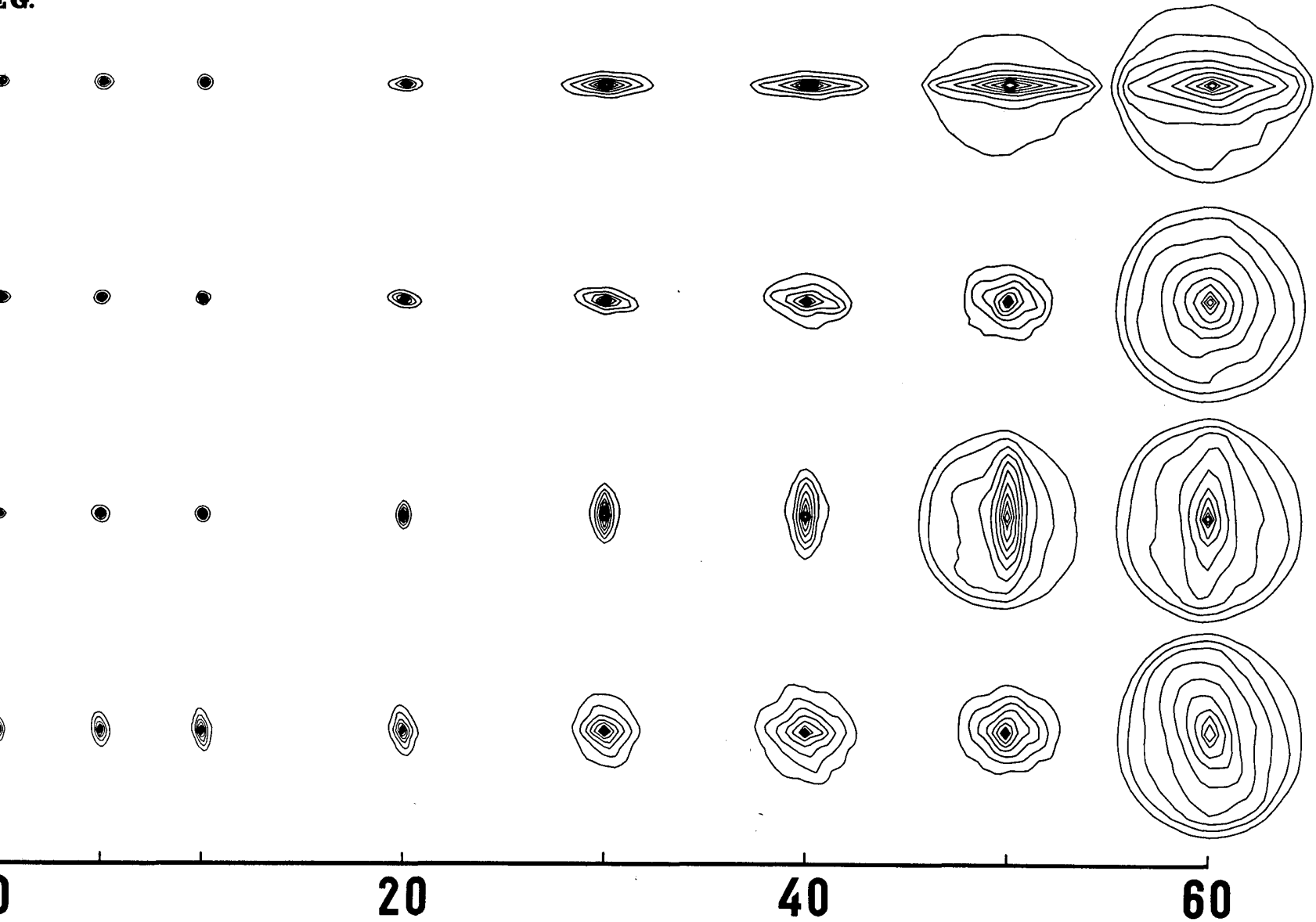

Fig. 2. Complete set of aerial PSF's for four subjects (SB, RN, PA, and GO). The axis represents retinal eccentricity in degrees (negative means nasal and positive means temporal). Each plot contains 10 contour curves with a linear interval of $10 \%$ of the peak value, ranging from $5 \%$ to $95 \%$ of that maximum value. At the top is a scale of the plots showing the extent of $1^{\circ}$ of visual field.

- SB, age 24 years, 0.2-D hyperopic, with small amount of residual (less than $0.12 \mathrm{D}$ ) direct astigmatism (horizontal)

- RN, 34 years, 0.2-D myopic, with $0.2 \mathrm{D}$ of direct astigmatism

- PA, 30 years, 0.75-D myopic, and practically astigmatism free

- GO, 25 years, with $0.5 \mathrm{D}$ of inverse astigmatism (vertical).

The MTF was measured in 15 retinal locations: the fovea and $5^{\circ}, 10^{\circ}, 20^{\circ}, 30^{\circ}, 40^{\circ}, 50^{\circ}$, and $60^{\circ}$ in both nasal and temporal retina along the horizontal meridian. However, the 15 measurements were completed only for subjects RN and GO, because, for large eccentricities (typically $50^{\circ}$ and $60^{\circ}$ on the nasal side) in the other two subjects, we often found artifacts in the aerial images, such as stripes and diffraction patterns, that were caused perhaps by spurious reflections from some ocular structure. To obtain the complete set of measurements we used three different zoom magnifications. We used a focal length of $200 \mathrm{~mm}$ from the fovea to $20^{\circ}, 100 \mathrm{~mm}$ from $30^{\circ}$ to $50^{\circ}$, and $60 \mathrm{~mm}$ for $60^{\circ}$ (also $50^{\circ}$ temporal retina for subjects SB and PA because of the large amount of astigmatism).

\section{RESULTS}

\section{A. Aerial Images}

Figure 2 shows the complete set of average aerial images obtained at each eccentricity. Each plot contains 10 contour curves, starting from a minimum level of $5 \%$ of the maximum, with an interval of $10 \%$. The scale bar at the top of the figure corresponds to $1^{\circ}$ of visual angle. Negative and positive eccentricities mean nasal and temporal hemiretinas, respectively. Each row contains the experimental results for a particular subject (SB, RN, PA, or GO).

The double-pass aerial PSF's show several interesting features, which appear more marked here than in the retinal point spreads or the MTF's, since the aerial images are affected twice by the aberrations. The main characteristic is that the optical quality decreases monotonically with eccentricity. Also, astigmatism appears to be the dominant (monochromatic) aberration in both the fovea and the periphery, while the contribution of other aberrations, such as coma or irregular aberrations, is not so clear. The relative absence of coma is consistent with the doublepass results of Jennings and Charman. ${ }^{18}$ In double-pass monochromatic measurements, astigmatism appears to be the most important aberration, while the contribution of comalike aberrations begins to be important for pupils $5 \mathrm{~mm}$ in diameter or larger. ${ }^{14,32}$ That astigmatism is 
the dominant monochromatic aberration has been confirmed in the fovea by the method of Arnulf et al. ${ }^{20}$ which permits dynamic recording of the aerial image with the help of an image intensifier. By using this method these authors studied the relationship between astigmatism and accommodation. For a single subject with free accommodation, they found the two foci and the circle of least confusion by changing the vergence of the point source (see Fig. 7 of Ref. 20). Also, Santamaría et $a l^{33}$ have shown that by placing a cylindrical trial lens behind the eye they could convert the elongated astigmatic PSF into a diamondshaped one. More recently, by using the same method, we were able to find the two foci and the circle of least confusion in different points in the periphery (from $10^{\circ}$ to $40^{\circ}$ ). ${ }^{12}$

Most of the aerial PSF's show either of the two possible shapes that are typical of astigmatism: elongated in one direction, when the retina is close to one of the two Sturm foci-tangential (see SB, temporal) or sagittal (see PA, temporal)-or diamond shaped (or circular), when the PSF is close to the circle of least confusion (see GO, $+40^{\circ}$ ). There is a tendency in our four subjects, which is more marked on the temporal side, to accommodate near one of the astigmatism foci across a wide visual field. The image quality is maximum (minimum spread) in the fovea in three of our four subjects. From there, the optical quality decays first very slowly, from $\pm 5^{\circ}$ to nearly $\pm 20^{\circ}$; then from $20^{\circ}$ to $50^{\circ}$ the optical quality becomes progressively worse, so that in the far periphery, $60^{\circ}$, the optical quality becomes quite poor.

Figure 2 also shows that the variability among these subjects is relatively small when compared with differences between eccentricities. At each eccentricity, astigmatism seems to be the major source of variability. It is well known that peripheral astigmatism ${ }^{15-17}$ increases strongly with eccentricity, the Sturm interval in diopters being roughly proportional to $\alpha^{1.5}$, where $\alpha$ is the visual angle. ${ }^{34}$ However, intersubject differences are explained more by differences in refractive state (focusing) than by the amount of astigmatism, which, with the exception of the fovea and small eccentricities, seems to be roughly the same for a given eccentricity. In other words, since our measurements have been taken without the use of any spherical or cylinder compensation, we have obtained, for each eccentricity, a haphazard sampling of the possible refractive states across the Sturm interval. The appearance of the aerial image suggests that the refractive state is sometimes close to the tangential focus (see SB, $40^{\circ}$ ), sometimes close to the sagittal focus (see PA, $40^{\circ}$ ), sometimes close to the circle of least confusion (see GO, $-30^{\circ}$ ), and sometimes in an intermediate state (see $R N,-40^{\circ}$, which is between the circle of least confusion and the tangential focus). An interesting feature is that the two subjects with nearly symmetric aerial images in the fovea (SB and PA) show the larger elongations in the periphery, while the two subjects with foveal astigmatism (RN and GO) show much less elongated images in the periphery. Subject GO provides a clear example of a vertical elongation from $0^{\circ}$ to $\pm 20^{\circ}$ and a diamond shape from $30^{\circ}$ to $60^{\circ}$.

\section{B. Two-Dimensional Modulation Transfer Functions}

Examples of two-dimensional MTF's are plotted in Fig. 3 for subject PA (fovea, $20^{\circ}, 40^{\circ}$, and $60^{\circ}$, in the temporal retina); MTF's averaged over the four subjects and the two hemiretinas are shown in Fig. 4. We averaged across nasal and temporal hemiretinas because our data did not show large differences between them, despite the known displacement of the optic axis from the fovea. The contour curves start at a minimum level of $5 \%$ of modulation, with a linear-level interval of $10 \%$. By comparing Figs. 3 and 4 we can see how the MTF's for subject PA are similar to but slightly better than the average; this subject is practically astigmatism free in the fovea, but the horizontally elongated peripheral MTF's for $20^{\circ}$ and $40^{\circ}$ suggest the presence of sagittal astigmatism, which was also observed in the aerial images. Figure 4 shows average data; the contour curves tend to show either a circular or a diamond shape (the shapes are clearer for large eccentricities) as a result of the averaging of three types of shape: tangential (vertical elongation in the MTF), sagittal (horizontal), and circle of least confusion (diamond). The monotonic decay in modulation and resolution with eccentricity is clearly visible.

\section{Strehl Ratio and Resolution}

The Strehl ratio can be computed as the volume under the MTF divided by the equivalent volume of a perfect (aberration-free) system with the same aperture. ${ }^{35}$ It provides a single parameter to measure the departure from the diffraction limit. The Strehl ratio has been computed for every MTF and is represented (as percent values) in Fig. 5 as a function of eccentricity. Symbols represent individual values, and the continuous curve corresponds to the average. It is clear that, for a 4-mm pupil, we are far from the diffraction limit. Even in the fovea the values are around $15 \%$, while the resolution of an optical system can be considered to be diffraction limited when the Strehl ratio is greater than $80 \%$ (Rayleigh criterion). This figure is a simple way to represent the decay of optical quality with eccentricity. The mean curve shows a bell shape, which could be roughly fitted to a Gaussian. The small but clear peak is centered in the fovea rather than on the optical axis (the peak typically falls approximately $5^{\circ}$ nasal). This is contrary to what one would expect on centered optical systems, in which the best image quality is always on axis. The absolute intersubject differences in optical quality are larger in the fovea and decrease with eccentricity. Although three of the four subjects show better optical quality in the fovea than in the near periphery, subject RN, with horizontal astigmatism in the fovea, shows better quality at $5^{\circ}$ and $10^{\circ}$ than in the fovea. The peak value for this subject is at $10^{\circ}$ temporal retina. We believe that this is an effect of astigmatism: it seems that foveal astigmatism could compensate for off-axis astigmatism at a particular retinal point if the two astigmatisms have the same value but opposite signs. For subject $\mathrm{RN}$, the foveal image has the maximum elongation (within $\pm 10^{\circ}$ ), while the minimum elongation (close to the circle of least confusion) is at $10^{\circ}$.

Figure 6 shows both a rough estimate of the retinal optical resolution as a function of eccentricity and a comparison with previous data of Jennings and Charman, ${ }^{18,19}$ in terms of widths at half-height of the aerial (external) double-pass image. The retinal resolution has been plotted on a log scale as a function of retinal eccentricity. It has been roughly estimated by the (commonly used) assumption of approximating point spreads to Gaussians, 


\section{P.A. FOVEA}

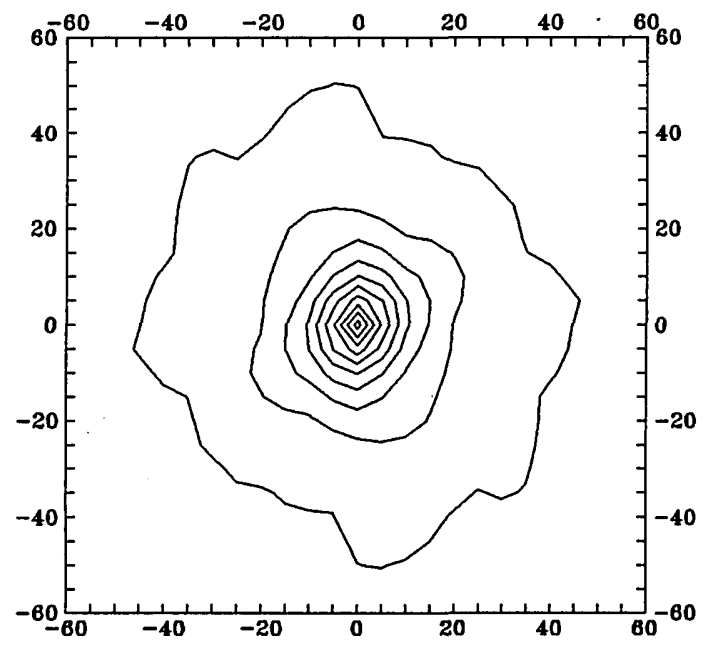

P.A. $40 \mathrm{deg} T$

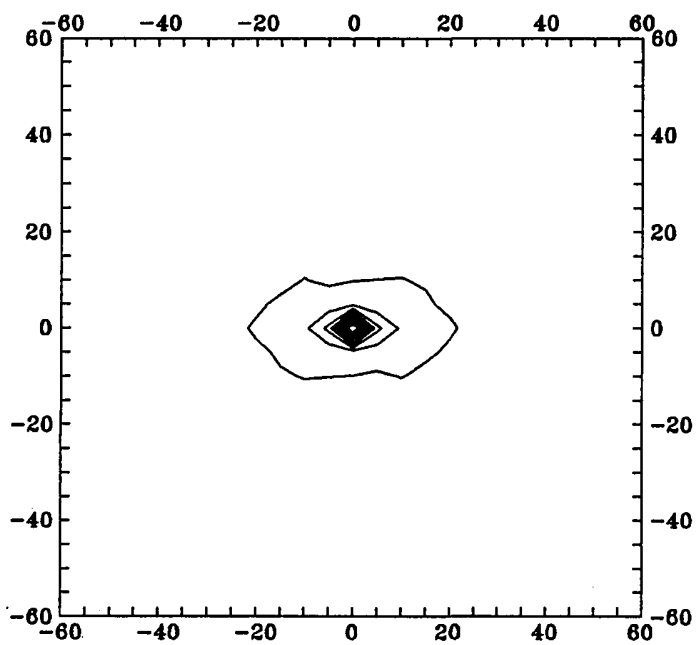

Fig. 3. Two-dimensional MTF's for subject PA in four eccentricities. Horizontal and vertical axes are in spatial-frequency coordinates (cycles per degree). Contour-curve levels are the same as in Fig. 2.

which means that the energy lost in the PSF peak with respect to the perfect system (the Strehl ratio) would be spread as a result of a two-dimensional Gaussian broadening. The value plotted is the full width at halfheight of this Gaussian. This approach holds within a reasonable approximation only when the optical quality is far from the diffraction limit, i.e., the Strehl ratio is low. Although there is no easy way with noisy double-pass data to obtain an accurate direct measure of retinal (singlepass) optical resolution and this method involves one assumption that is to some extent arbitrary, the results could be a reasonable estimate of the actual optical resolution. The results are shown in Fig. 6 as filled circles on the continuous curve. The average resolution that we have estimated with a 4-mm pupil diameter is approximately $0.8^{\prime}$ in the fovea, $1^{\prime}$ at $20^{\circ}, 2^{\prime}$ at $40^{\circ}$, and $4.5^{\prime}$ at $60^{\circ}$.

Figure 6 also compares the present results with those of Jennings and Charman. ${ }^{18,19}$ The open triangles corre-
P.A. 20 deg T

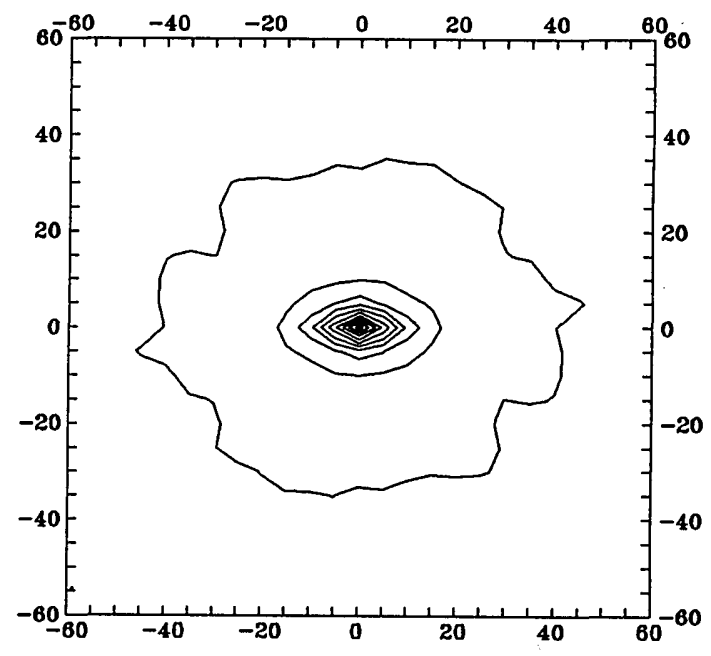

P.A. $60 \mathrm{deg} T$

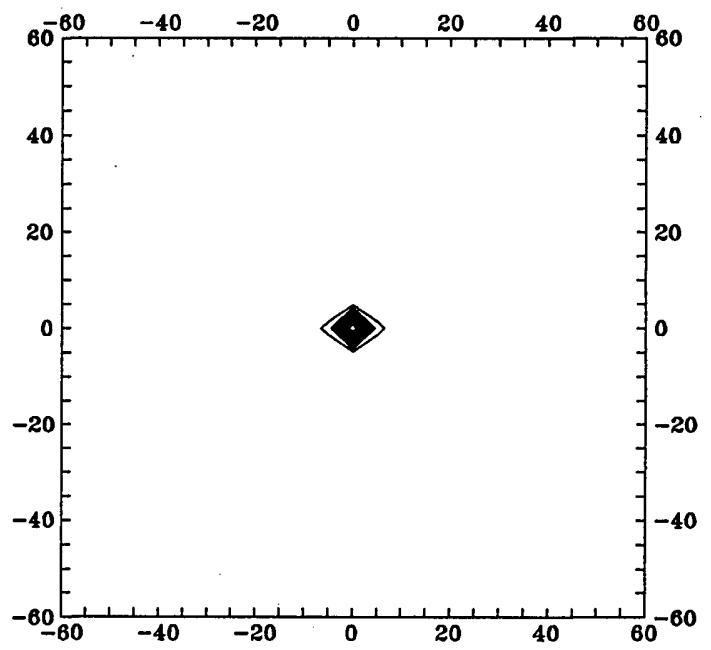

spond to an experiment ${ }^{19}$ performed with conditions similar to ours (4-mm pupil, 3-D vergence, and free accommodation). The plotted values are the square root of the product of the horizontal and vertical full width at half-height of the aerial images (line spreads external to the eye). Our results are shown as filled triangles (resolution has been estimated with the same method, but now from horizontal and vertical slices of the aerial PSF). Open stars show data (half-widths) obtained with paralyzed accommodation and 7.5-mm natural pupil. ${ }^{18}$ Comparison of our data with those of Jennings and Charman is complicated by the fact that we measured point spreads while they measured line spreads. Although horizontal or vertical slices of the PSF are not exactly equivalent to those of the line spreads, their widths are roughly similar (the equivalence holds in the Fourier domain between onedimensional MTF's computed from line-spread functions and slices of the two-dimensional MTF). In any case, 
both sets of data from Jennings and Charman show much poorer optical quality than our data at all retinal eccentricities, including the fovea. In the case that is most comparable with ours, with a 4-mm pupil and free accomFOVEA

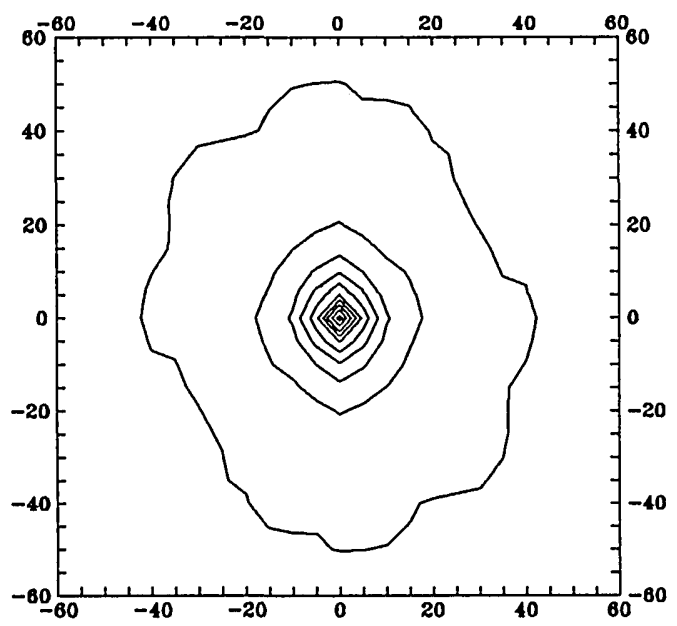

$20 \mathrm{deg}$

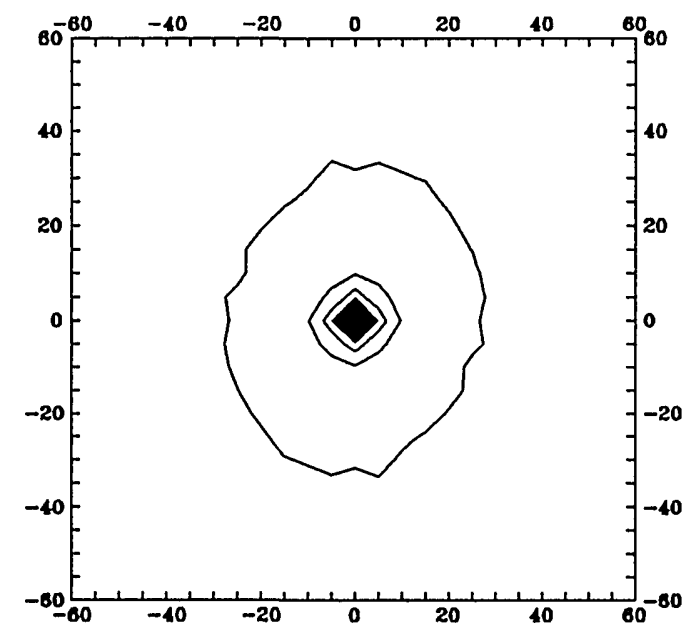

$40 \mathrm{deg}$

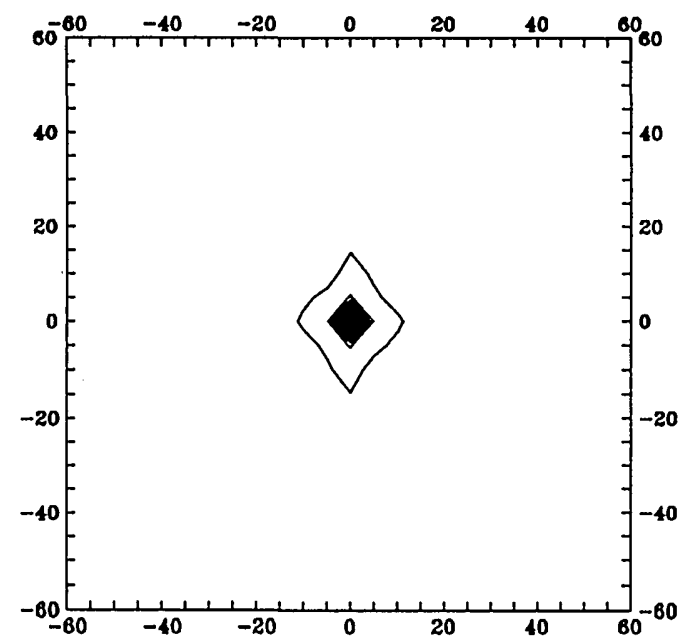

modation, Jeninings and Charman attributed the low optical quality that they obtained to lags in accommodation. In our experiment both short-exposure imaging and binocular viewing seemed to help to eliminate almost com-

$10 \mathrm{deg}$

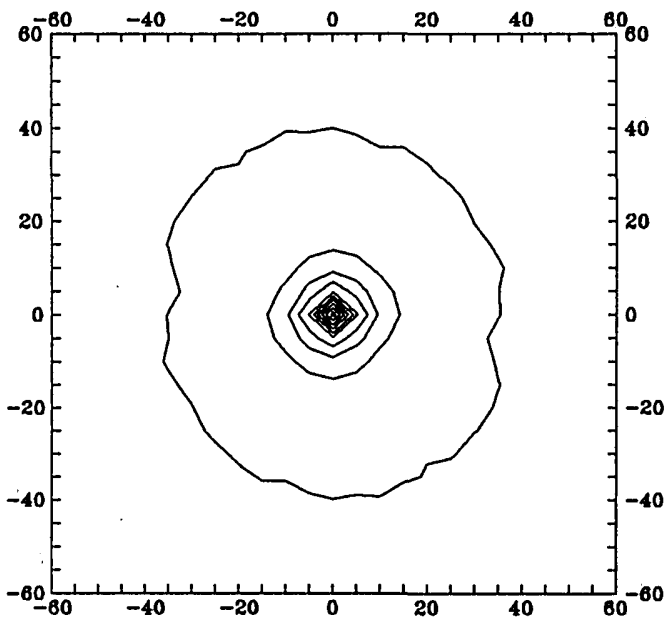

$30 \mathrm{deg}$

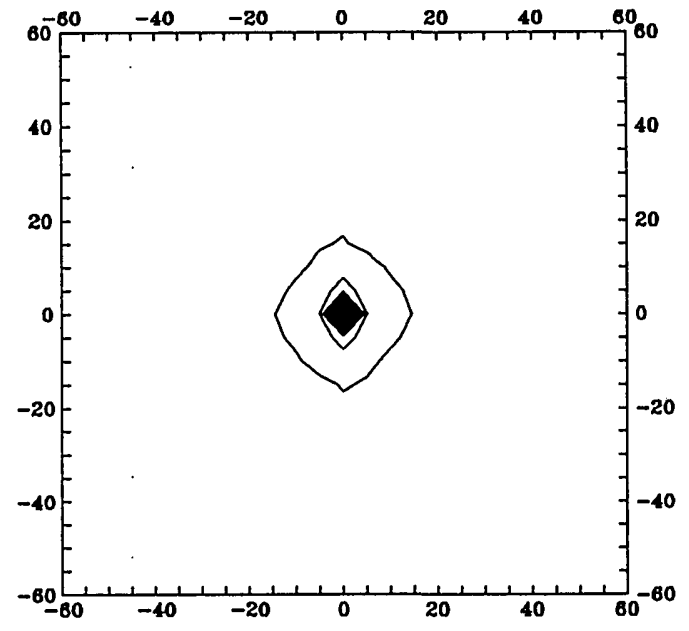

$60 \mathrm{deg}$

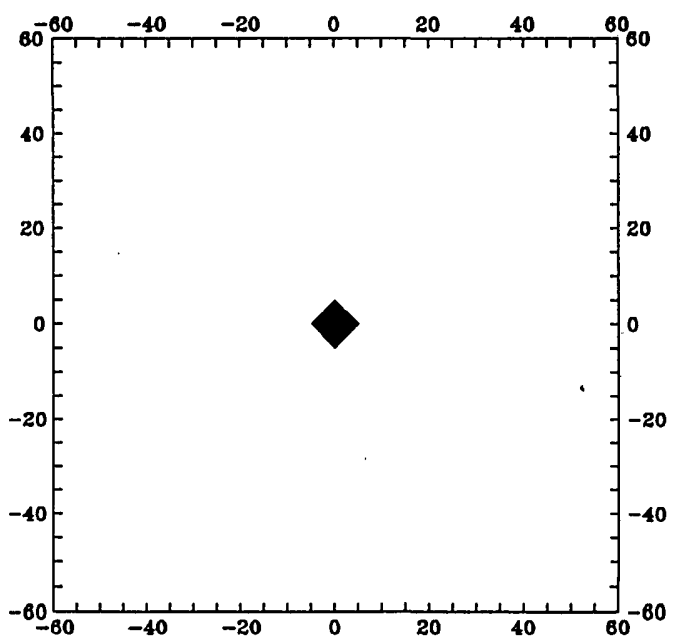

Fig. 4. Average MTF's at six eccentricities. The average has been taken over four subjects and two hemiretinas. The resulting contour curves tend to show either a diamond or a circular shape. 


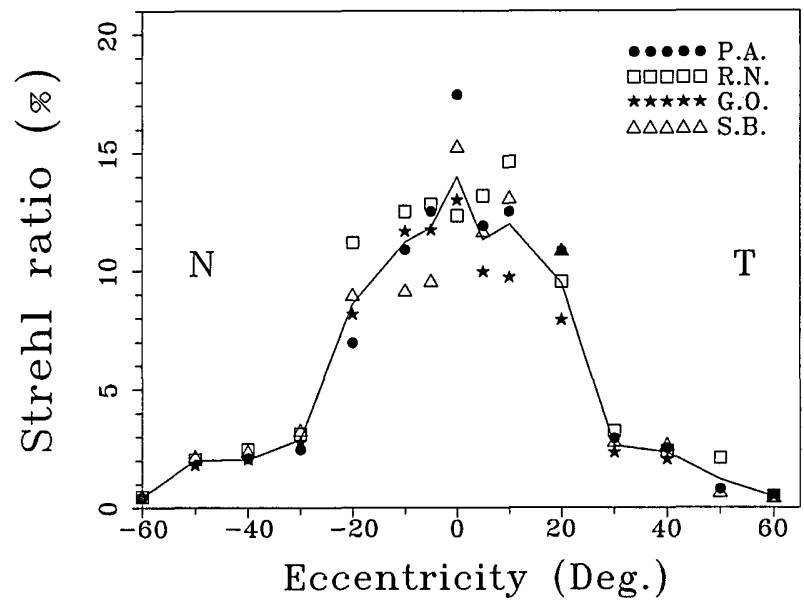

Fig. 5. Percent Strehl ratios computed for the complete set of data. Symbols represent individual values; the continuous curve represents the average.

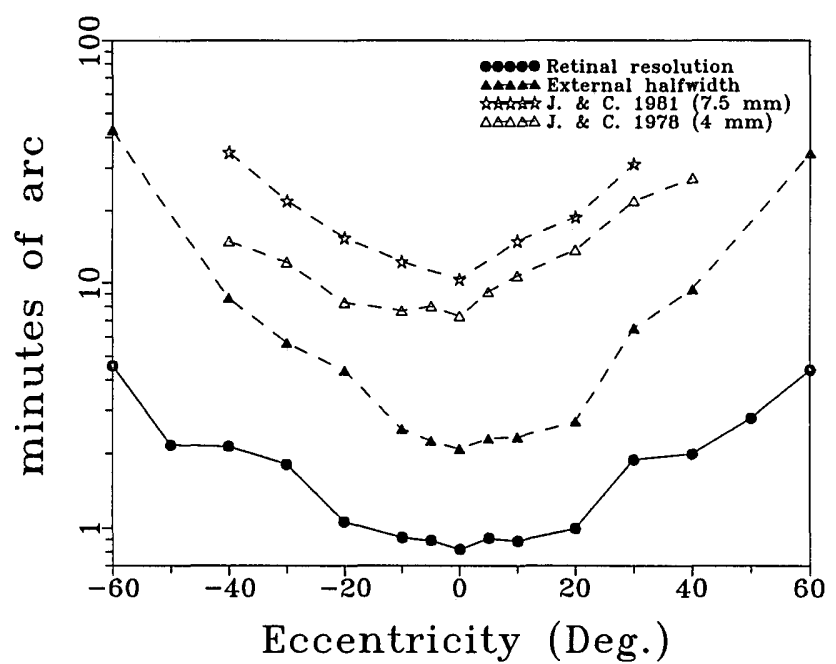

Fig. 6. Comparison with data from Jennings and Charman. The open symbols - triangles and stars-represent widths at halfheight of the aerial line-spread functions from Jennings and Charman for 4-mm-pupil free accommodation ${ }^{19}$ and 7.5-mm-pupil paralized accommodation, ${ }^{18}$ respectively. Filled triangles correspond to our data. A rough estimate of retinal optical resolution, computed from the Strehl ratio, is also included (filled circles with continuous curve). All sets of data are plotted versus retinal eccentricity, in units of arcmin on a log scale.

pletely the possibility of that kind of problem. The data of Jennings and Charman, with a 7.5-mm pupil and paralyzed accommodation, also show reduced optical quality, even though the data were obtained with an optimum refractive compensation. In this case the optical degradation that they observed may have been due to the effects of the large aberrations that are usually associated with large pupils. Furthermore, they used white light for their experiments, whereas we used monochromatic light; therefore one might expect their aerial LSF's to be somewhat broader than ours as a result of chromatic aberration.

\section{Signal-to-Noise Ratio}

To provide an estimate of the signal-to-noise ratio (SNR) in our MTF's, we have obtained error bars by computing four different estimates of each MTF from four partial averages, each containing eight exposures (see Subsec- tion 3.C). This method yields an overestimation of the MTF, which can be significant at high frequencies as a result of speckle noise. This effect can be seen in Fig. 7, where the center of the error bars tends sometimes to be placed above the MTF curve. Nonetheless, it provides a good estimate of the experimental variability. Two typical examples are shown in Fig. 7, which correspond to vertical cuts in the fovea (higher-quality, nearly astigmatism-free case) and $30^{\circ}$ temporal (poor quality, strong astigmatism) for subject PA. The length of the bars represents \pm 1 standard error of the mean. Variability is a little larger in the fovea, where microfluctuations of accommodation can have a somewhat larger effect. The MTF curves always show two parts: one part is for low frequencies with a larger slope and SNR $\approx 10-20$ (typically for modulations greater than $10 \%$ ); the second part is for high frequencies and is flatter, with a lower SNR that stays relatively constant with respect to the larger frequencies that are represented in Fig. 7 (this constancy can be observed in the figure as a roughly constant size of the error bars in the logarithmic scale). The SNR's for the two corresponding curves of Fig. 6 have been estimated from the error bars and are for the midhigh-frequency range of this figure approximately $\mathrm{SNR} \approx$ 4 and SNR $\approx 5$ for the fovea and $30^{\circ}$, respectively.

\section{E. Radial Profiles}

The aim of the present study was to provide estimates of the monochromatic image quality of the eye for stimuli at a fixed distance from the eye, viewed with natural accommodation and without correction of possible defocus or astigmatism. Mainly because of astigmatism, image contrast depends on the orientation of a given spatialfrequency component of the object. Furthermore, the orientation that yielded the best and worst contrast varied from observer to observer because of individual differences in the amount (and axis) of astigmatism and refractive state, which were not systematically controlled.

Keeping these facts in mind, and in order to go further in the analysis of the MTF data that are summarized in Figs. 3 and 4, we have extracted one-dimensional data sets

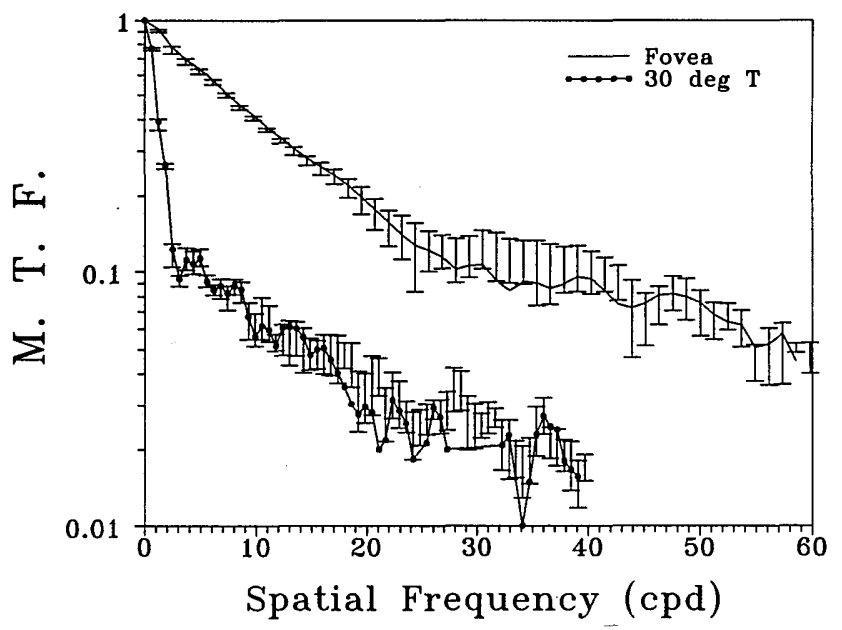

Fig. 7. Two examples, from data for subject PA, illustrating typical measuring errors. Two vertical cuts of two-dimensional MTF's are plotted on a log scale, the upper curve corresponding to the fovea in an astigmatism-free direction and the lower curve corresponding to $30^{\circ}$ of eccentricity with strong astigmatism. The bars represent the standard error of the mean. 


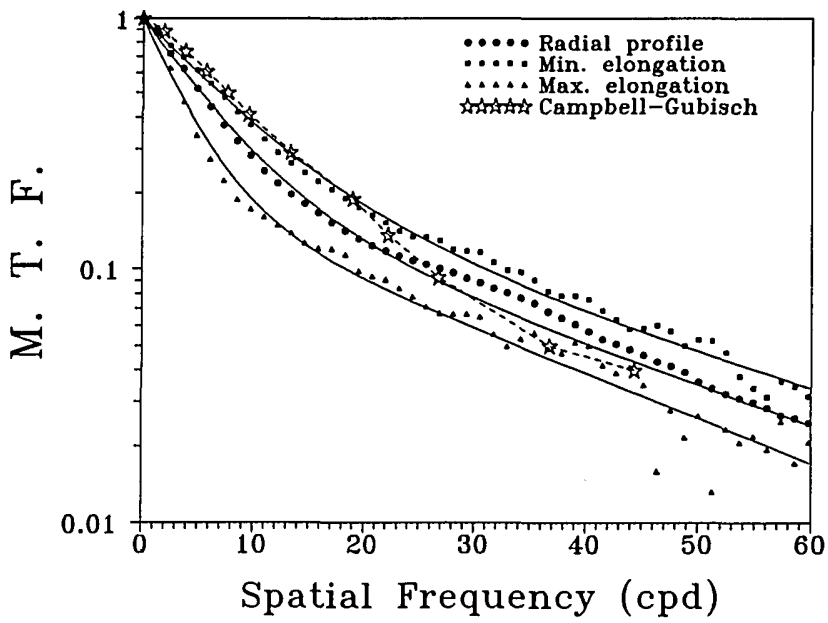

Fig. 8. Foveal data (filled symbols) and the results of curve fitting to the sum of two exponentials (continuous curves). Three experimental sets of MTF data are plotted on a logarithmic scale: average radial profile (circles), upper (minimum elongation, squares) bounds, and lower (maximum elongation, triangles) bounds. Data from Campbell and Gubisch ${ }^{9}$ for a $3.8-\mathrm{mm}$ pupil (open stars) are also included.

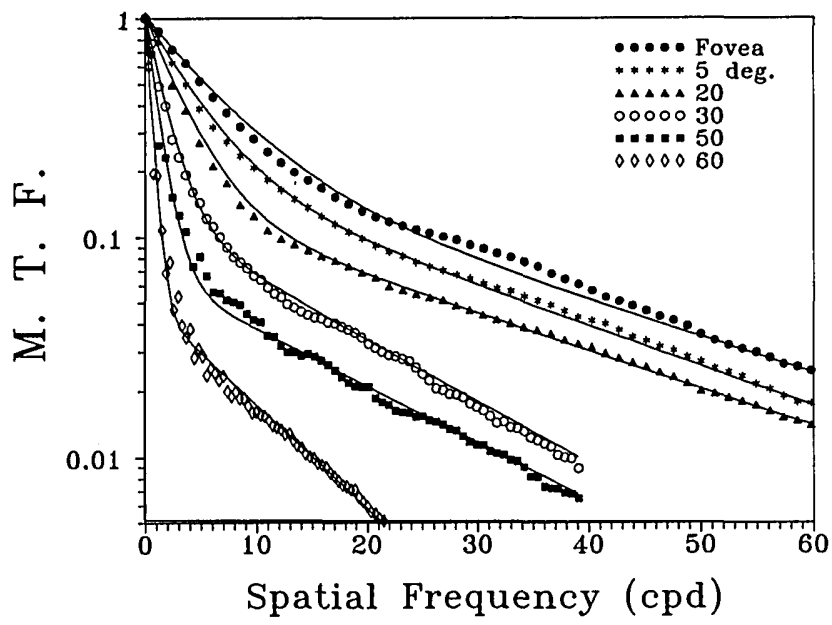

Fig. 9. Average radial profiles (symbols) and results of curve fitting (continuous curves) for six eccentricities. Results for $10^{\circ}$ and $40^{\circ}$ have been left out for the sake of clarity.

for each eccentricity. One set is the radial profile of the mean two-dimensional MTF from Fig. 4, defined as the average over all orientations. Because of the tendency of the mean MTF's to show rotational symmetry, the radial profiles provide a good description of the average MTF. We also have computed standard deviations and upperand lower-bound values of the MTF's at each eccentricity.

The average foveal radial profile and upper-bound and lower-bound cases are plotted in Fig. 8 on a logarithmic scale, along with the results of a least-squares fit shown as continuous curves (see below). The reason that we include upper and lower bounds is that they show the minimum and the maximum effect of astigmatism: the lower curve corresponds approximately to the subject and the orientation that show the maximum elongation of the aerial image, while the upper curve corresponds to the minimum elongation. Roughly speaking, this would represent an astigmatism-free MTF. The average MTF obtained by Campbell and Gubisch ${ }^{9}$ for a 3.8-mm pupil is also included (open stars). In the low-frequency range (up to $20 \mathrm{cpd}$ ) their data follow our upper-bound case, which might be expected since they used optimum refractive compensation (sphere and cylinder). For higher frequencies their MTF declines below our radial profile. One possible explanation for this discrepancy could be that they used white light (a xenon lamp), whereas our MTF's are monochromatic (He-Ne laser) and therefore our data are not affected by chromatic aberration.

The results of curve fitting are also included in Fig. 8; we have fitted the sum of two exponential functions: one with higher weight and slope to fit the low-frequency range, the second to fit the high-frequency part:

$$
\operatorname{MTF}(f)=(1-C) \exp (-A f)+C \exp (-B f)
$$

where $A, B$, and $C$ are the free-fitting parameters and $f$ is the spatial frequency in cycles per degree. The same theoretical function has been used to fit all sets of data.

The average radial profiles for the different eccentricities are summarized in Fig. 9. The symbols represent the experimental data and the curves that we have obtained by the nonlinear least-squares-fitting procedure described above. Two eccentricities, $10^{\circ}$ and $40^{\circ}$, have been left out for the sake of clarity: the first is nearly the same as $5^{\circ}$, and the second lies between $30^{\circ}$ and $50^{\circ}$, very close to $50^{\circ}$. The radial profiles show a progressive

Table 1. Best Least-Squares Fit (Exponential Plus Exponential) to the Radial Profiles ${ }^{a}$

\begin{tabular}{cllll}
\hline$\theta$ & $A(\theta)$ & $B(\theta)$ & $C(\theta)$ & \multicolumn{1}{c}{ Error } \\
\hline Fovea & 0.172 & 0.037 & 0.22 & $1.3 \times 10^{-3}$ \\
$5^{\circ}$ & 0.245 & 0.041 & 0.2 & $1.4 \times 10^{-3}$ \\
$10^{\circ}$ & 0.245 & 0.041 & 0.2 & $2.3 \times 10^{-3}$ \\
$20^{\circ}$ & 0.328 & 0.038 & 0.14 & $2.9 \times 10^{-3}$ \\
$30^{\circ}$ & 0.606 & 0.064 & 0.12 & $3.2 \times 10^{-3}$ \\
$40^{\circ}$ & 0.82 & 0.064 & 0.09 & $7.8 \times 10^{-3}$ \\
$50^{\circ}$ & 0.93 & 0.059 & 0.067 & $1.24 \times 10^{-2}$ \\
$60^{\circ}$ & 1.89 & 0.108 & 0.05 & $1.13 \times 10^{-2}$ \\
\hline
\end{tabular}

${ }^{a} f$, Spatial frequency; $\theta$, retinal eccentricity. $\operatorname{MTF}_{\mathrm{rp}}(f)=(1-C)$ $\exp (-A f)+C \exp (-B f)$.

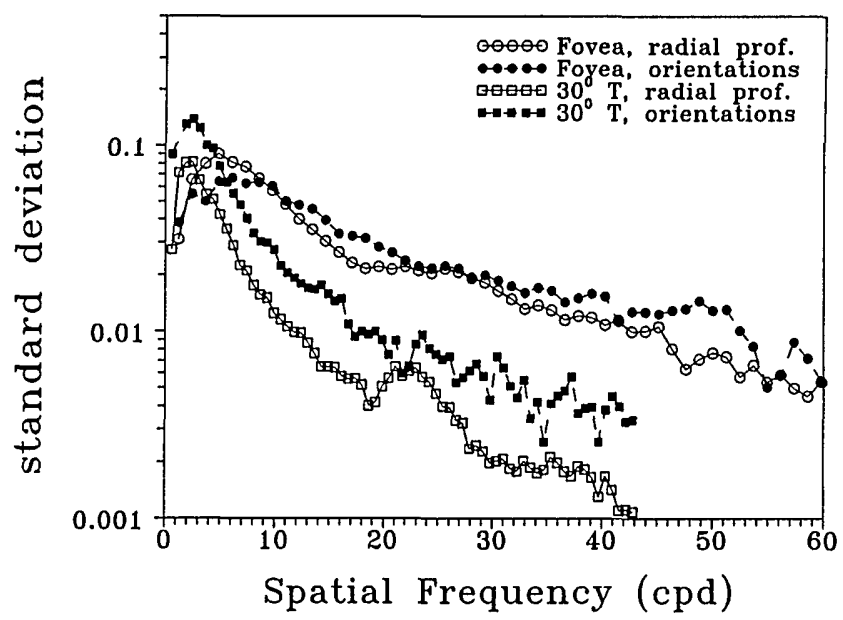

Fig. 10. Four examples of SD's showing interorientation (filled symbols) and intersubject (open symbols) variabilities of the MTF in the fovea (circles) and in the periphery $-30^{\circ}$ temporal retina(squares). SD's are in modulation-increment units, plotted on a logarithmic scale as a function of spatial frequency. 
Table 2. Modulation Transfer as a Function of Retinal Eccentricity $(\theta)$ and Spatial Frequency $(f)^{a}$

\begin{tabular}{lcccccr}
\hline & \multicolumn{7}{c}{ Parameter } \\
\cline { 2 - 7 } & $A_{1}$ & $A_{2}$ & $B_{1}$ & $B_{2}$ & $C_{1}$ & $C_{2}$ \\
\hline Radial profile & 0.1743 & 0.0392 & 0.0362 & 0.0172 & 0.215 & 0.00294 \\
Upper-bound case & 0.1093 & 0.0359 & 0.0279 & 0.0187 & 0.223 & 0.00296 \\
Lower-bound case & 0.2292 & 0.0351 & 0.0304 & 0.0186 & 0.171 & 0.00224 \\
\hline
\end{tabular}

${ }^{a}$ Theoretical function and parameters were obtained from radial profiles and upper-bound and lower-bound cases. $\quad M(\theta, f)=\left(1-C_{1}+C_{2} \theta\right)$ $\exp \left[-A_{1} \exp \left(A_{2} \theta\right) f\right]+\left(C_{1}-C_{2} \theta\right) \exp \left[-B_{1} \exp \left(B_{2} \theta\right) f\right]$.

and quite regular monotonic decay with eccentricity. Table 1 shows the resulting parameters and the rms fitting errors.

The sum of two exponentials appears to be an efficient parameterization for the entire family of curves. It uses only three parameters for each curve and provides a good fit to the complete data set. This would permit, in principle, a fast, direct parametric estimation of the radial profile of the retinal PSF, since the two-dimensional Fourier transform of the sum of two exponentials is the sum of two Lorentzians. ${ }^{36}$ However, the parametric MTF's yield PSF's that are too narrow because the exponentials never reach zero, so the PSF's fail to fit the actual cutoff frequency. Consequently, one should not extrapolate the curve fitting beyond the limits shown in the figures or use them to estimate point spreads.

\section{F. Variability}

We were interested in comparing the partial effects of astigmatism and the effects of intersubject differences. Therefore two different standard deviations (SD's) were computed at each eccentricity, as shown in Fig. 10. One is the intersubject SD computed from the radial profiles (open symbols). The second SD (filled symbols) was computed as the average (over the four subjects) of the interorientation SD's, computed from one-dimensional MTF profiles corresponding to the four main orientations (horizontal, vertical, $45^{\circ}$, and $135^{\circ}$ ). In Fig. 10 the two types of SD are plotted for the foveal (circles) and the $30^{\circ}$ temporal (squares) cases in units of modulation increment, as a function of spatial frequency. In both cases the mean interorientation variability (filled symbols) is larger than the intersubject variability (open symbols), the difference being small in the fovea but larger in the periphery, where astigmatism is more important. This result suggests that astigmatism, which is the main cause of orientation variability, is also the major cause of overall variability. The pattern of decline of the SD with spatial frequency and eccentricity resembles that of the MTF's, suggesting a sort of rough proportionality.

\section{G. Modulation Transfer As a Function of Eccentricity}

An additional fitting procedure, applied to the set of theoretical curves of Fig. 9, allows the entire family of curves to be represented by a single theoretical function of two variables: spatial frequency and eccentricity. Each set of the three parameters of Eq. 1, which are listed in Table 1, has been fitted to a theoretical function of eccentricity: exponentials again for parameters $A$ and $B$ and linear function for parameter $C$. The final theoretical curve and the set of parameters are listed in Table 2, which also includes the result of applying the same fitting procedure to the upper and lower bounds (as was shown in Fig. 8 for the foveal case). We point out that, whereas we have obtained a good fit with the radial profiles, the fitting results for the upper- and lower-bound data are not so good. The upper-bound MTF's, which are less affected by astigmatism, appear to be nearly constant between $5^{\circ}$ and

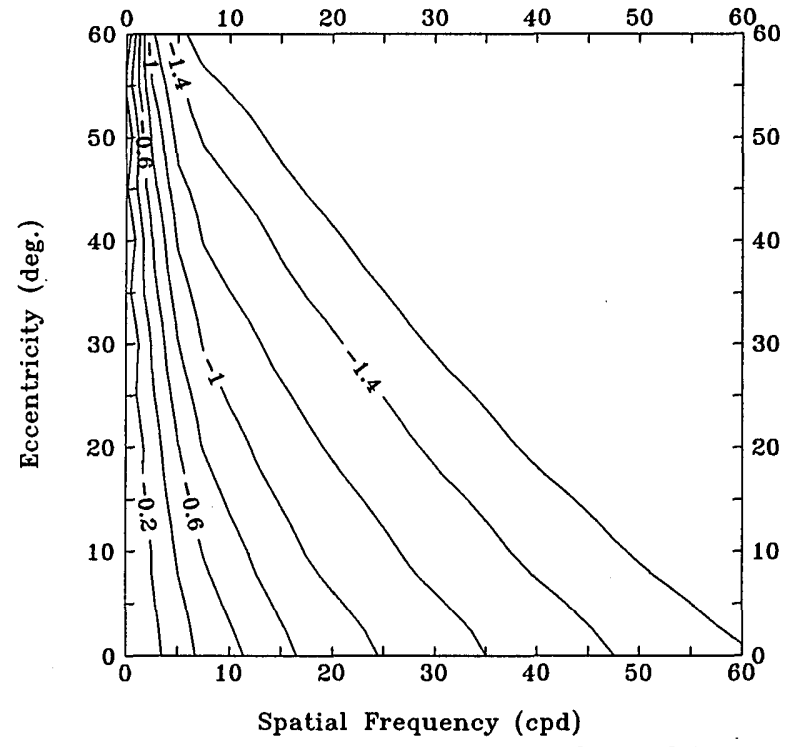

Fig. 11. Modulation transfer as a function of spatial frequency and eccentricity. This figure represents average data (radial profiles). The contour curves are on a $\log _{10}$ scale, and the spacing is $0.2 \log$ unit.

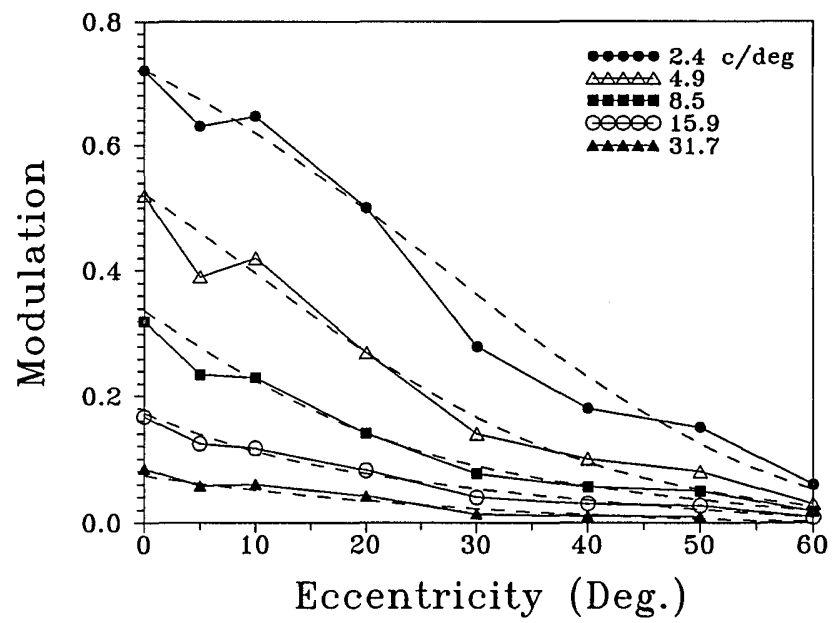

Fig. 12. Modulation as a function of retinal eccentricity for five different spatial frequencies. Filled symbols and continuous curves represent average experimental data (radial profiles); open symbols and dashed curves show the resulting modulation values computed from the theoretical two-dimensional function presented in Table 2. 
$20^{\circ}$ and almost as good as the foveal radial profile. This is in agreement with what was found in a psychophysical experiment by Still et $a l^{37}$

The resulting theoretical modulation transfer (radial profiles) as a function of spatial frequency and eccentricity is shown as a contour plot in Fig. 11. The contour curves correspond to a logarithmic scale. Figure 12 represents the average modulation as a function of eccentricity for five different spatial frequencies. Filled symbols and solid curves represent experimental data, while open symbols and dashed curves have been obtained as one-dimensional slices of the theoretical function from Table 2 and Fig. 11. The agreement is good if we take into account the small number of parameters that were used to fit the large amount of experimental data. This kind of representation can be useful, as it shows the pattern of decay of the average modulation with eccentricity.

\section{CONCLUSIONS}

The off-axis image quality has been found to be better than was previously thought. The overall image quality of the eye as a function of eccentricity resembles that of a wide-angle lens; i.e., the optical quality in the fovea is not particularly good when compared with conventional optical systems, but it shows relatively good off-axis behavior, maintaining relatively constant quality with eccentricity over a wide visual field. Only the far periphery shows a large decline in image quality.

\section{ACKNOWLEDGMENTS}

This project has been supported by the Comisión Interministerial de Ciencia y Tecnología (Spain) under grant PRONTIC P18-88/88-0198 to R. Navarro and also by grants EY01319, EY04367, and AFOSR 85-0019 to D. Williams. We thank subjects Gloria Oller and Santiago Baña for their kind collaboration.

\section{REFERENCES}

1. A. Ivanoff, Les Aberrations de l'Oeil (Editions de la Revue d'Optique Théorique et Instrumentale, Paris, 1953).

2. M. F. Flamant, "Etude de la repartition de lumière dans l'image rétinienne d'une fente," Rev. Opt. 34, 433-459 (1955).

3. G. Westheimer and F. W. Campbell, "Light distribution in the image formed by the living human eye," J. Opt. Soc. Am. 52, 1040-1045 (1962).

4. J. Krauskopf, "Light distribution in human retinal images," J. Opt. Soc. Am. 52, 1046-1050 (1962).

5. J. Santamaría, P. Artal, and J. Bescós, "Determination of the point-spread function of human eyes using a hybrid opticaldigital method," J. Opt. Soc. Am. A 4, 1109-1114 (1987).

6. P. Artal and R. Navarro, "Simultaneous measurement of two point-spread functions at different locations across the human fovea," Appl. Opt. 31, 3646-3656 (1992).

7. A. Arnulf and O. Dupuy, "La transmission des contrastes par le système optique de l'oeil et les seuils de contrastes rétiniennes," C. R. Acad. Sci. (Paris) 250, 2757-2759 (1960).

8. F. W. Campbell and D. G. Green, "Optical and retinal factors affecting visual resolution," J. Physiol. (London) 181, 576593 (1965).

9. F. W. Campbell and R. W. Gubisch, "Optical image quality of the human eye," J. Physiol. (London) 186, 558-578 (1966).

10. R. Röhler, U. Miller, and M. Aberl, "Zur Messung der Modulationsübertragungsfunktion des lebenden menschlichen Auges im reflektierten Licht," Vision Res. 9, 407-428 (1969).
11. W. N. Charman and J. A. M. Jennings, "The optical quality of the monochromatic retinal image as a function of focus," Br. J. Physiol. Opt. 31, 119-134 (1976).

12. P. Artal, R. Navarro, D. Brainard, S. Galvin, and D. R. Williams, "Off-axis optical quality of the eye and retinal sampling," Invest. Ophthalmol. Vis. Sci. Suppl. 33, 1342 (1992).

13. G. Walsh, W. N. Charman, and H. C. Howland, "Objective technique for the determination of monochromatic aberrations of the human eye," J. Opt. Soc. Am. A 1, 987-992 (1984).

14. P. Artal, "Calculations of two-dimensional foveal retinal images in real eyes," J. Opt. Soc. Am. A 7, 1374-1381 (1990).

15. C. E. Ferree, G. Rand, and C. Hardy, "Refraction for the peripheral field of vision," Arch. Ophthalmol. 5, 717-731 (1931).

16. C. E. Ferree and G. Rand, "Interpretation of refractive conditions in the peripheral field of vision: a further study," Arch. Ophthalmol. 9, 925-938 (1933).

17. F. Rempt, J. Hoogerheide, and W. P. H. Hoogenboom, "Peripheral retinoscopy and the skiagram," Ophthalmologica 162, 1-10 (1971).

18. J. A. M. Jennings and W. N. Charman, "Off-axis image quality in the human eye," Vision Res. 21, 445-454 (1981).

19. J. A. M. Jennings and W. N. Charman, "Optical image quality in the peripheral retina," Am. J. Optom. Physiol. Opt. 55, 582-590 (1978).

20. A. Arnulf, J. Santamaría, and J. Bescós, "A cinematographic method for the dynamic study of the image formation by the human eye. Microfluctuations of the accommodation," J. Opt. (Paris) 12, 123-128 (1981).

21. W. N. Charman and J. A. M. Jennings, "Objective measurements of the longitudinal chromatic aberration of the human eye," Vision Res. 16, 999-1005 (1976).

22. Y. U. Ogboso and H. E. Bedell, "Magnitude of lateral chromatic aberration across the retina of the human eye," J. Opt. Soc. Am. A 4, 1666-1672 (1987).

23. L. N. Thibos, A. Bradley, D. L. Still, X. Zhang, and P. A. Howarth, "Theory and measurement of ocular chromatic aberration," Vision Res. 30, 33-49 (1990).

24. D. Sliney and M. Wolbarsht, Safety With Lasers and Other Optical Sources (Plenum, New York, 1980).

25. F. C. Delori and K. P. Pflibsen, "Spectral reflectance of the human ocular fundus," Appl. Opt. 28, 1061-1077 (1989).

26. G. J. van Blokland, "Directionality and alignment of the foveal receptors, assessed with light scattered from the human fundus in vivo," Vision Res. 26, 495-500 (1986).

27. J. M. Gorrand, "Reflection characteristics of the human fovea assessed by reflecto-modulometry," Ophthalmol. Physiol. Opt. 9, 53-60 (1989).

28. J. J. Vos, J. Walraven, and A. van Meeteren, "Light profiles of the foveal image of a point source," Vision Res. 16, 215-219 (1976).

29. R. Navarro, "Incorporation of intraocular scattering in schematic eye models," J. Opt. Soc. Am. A 2, 1891-1894 (1985).

30. J. F. Simon and P. M. Denieul, "Influence of the size of test field employed in measurements of the modulation transfer function of the eye," J. Opt. Soc. Am. 63, 894-896 (1973).

31. P. Artal, J. Santamaría, and J. Bescós, "Phase-transfer function of the human eye and its influence on point-spread function and wave aberration," J. Opt. Soc. Am. A 5, 1791-1795 (1988).

32. P. Artal, J. Santamaría, and J. Bescós, "Retrieval of wave aberration of human eyes from actual point-spread function data," J. Opt. Soc. Am. A 5, 1201-1206 (1988).

33. J. Santamaría, A. Plaza, and J. Bescós, "Dynamic recording of the binocular point spread function of the eye optical system," Opt. Pura Apl. (Madrid) 17, 57-63 (1984).

34. W. Lotmar and T. Lotmar, "Peripheral astigmatism in the human eye: experimental data and theoretical model predictions," J. Opt. Soc. Am. 64, 510-513 (1974).

35. W. Smith, Modern Optical Engineering (McGraw-Hill, New York, 1966).

36. R. N. Bracewell, The Fourier Transform and Its Applications (McGraw-Fill, New York, 1978).

37. D. L. Still, L. N. Thibos, and A. Bradley, "Peripheral image quality is almost as good as central image quality," Invest. Opththalmol. Vis. Sci. Suppl. 30, 52 (1989). 